\title{
LA ESCUELA DE ARTES Y OFICIOS UN INSTRUMENTO PARA EL DESARROLLO SINÉRGICO*
}

Jemay Mosquera Téllez**

Celesky Mariel Reyes Caballero****

Grupo de Investigación Gestión Integral del Territorio - GIT

Universidad de Pamplona N.S.

Artículo de investigación. Hace parte de los procesos que se adelantan en el grupo de investigación Gestión Integral del Territorio - GIT de la Universidad de Pamplona, en el marco del proyecto de investigación "Parque Temático Pueblito Nortesantandereano" $y$ en el desarrollo del trabajo de grado del programa de arquitectura "Escuela de Artes y Oficios del Parque Temático Pueblito Nortesantandereano - Pamplona" realizado por Celesky Mariel Reyes Caballero en el año 2011 .

\section{RESUMEN}

El proceso de caracterización y análisis de las artes y oficios en el departamento de Norte de Santander, posibilitó la identificación de los referentes culturales y los factores afines al entorno que inciden en el diseño de una escuela de artes y oficios y que, articulados a su proceso proyectual, sirven de base para valorar, recuperar y fortalecer los saberes artísticos, artesanales y técnicos locales, como prerrequisito para la búsqueda de un desarrollo sinérgico del municipio de Pamplona.

\section{PALABRAS CLAVE}

Artes, Diseño, Oficios, Saberes, Desarrollo sinérgico 


\section{ARTS AND CRAFTS SCHOOL, AN INSTRUMENT FOR SYNERGETIC DEVELOPMENT}

Jemay Mosquera Téllez***

Celesky Mariel Reyes Caballero****

Grupo de Investigación Gestión Integral del Territorio - GIT

Universidad de Pamplona N.S.
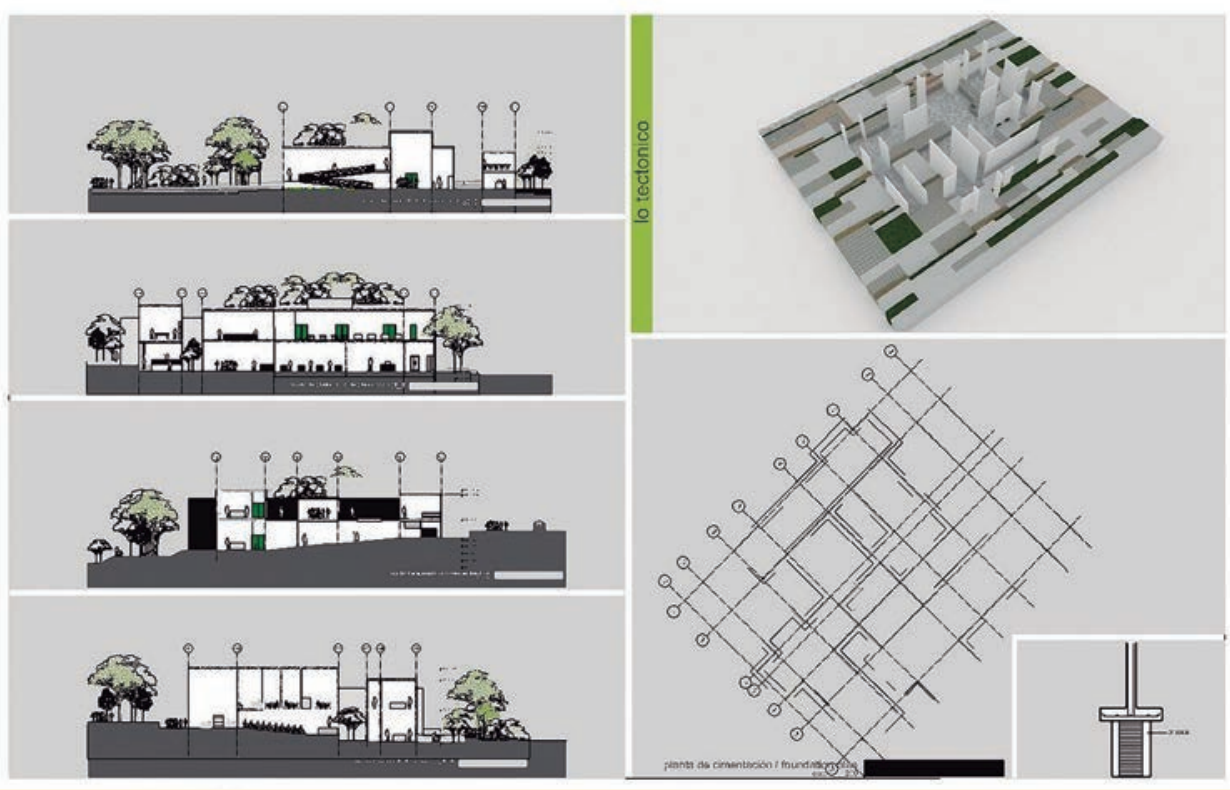

\section{ABSTRACT}

The process of characterization and analysis of arts and crafts in the department of Norte de Santander, enabled the identification of cultural references and the environment related factors that influence the design of a school of arts and crafts and, linked to their projective process, provide a basis to assess, restore and strengthen the local artistic, craft and technical knowledge, as a prerequisite to the search for synergistic development of the municipality of Pamplona.

\section{KEY WORDS}

Arts, Design, Crafts, Knowledge, Synergistic development
* Doctor en Arquitectura con énfasis en Planificación Urbana y Regional de la Academia de Ingeniería Municipal de Kharkiv, Ucrania (1997) y Arquitecto del Instituto de Ingenieros de la Construcción Comunal de Kharkiv, Ucrania (1988). Consultor de la Sociedad Colombiana de Arquitectos Regional Quindío (2002-2005). Profesor universitario en pregrados y/o posgrados de diferentes universidades colombianas desde 1997. Profesor Asistente, Director del Departamento de Arquitectura y Diseño Industrial (2005-2010) y Líder del Grupo de Investigación Gestión Integral del Territorio - GIT (2003-a la fecha) de la Universidad de Pamplona.

*** Arquitecta de la Universidad de Pamplona (20I I) con capacidades en el desempeño de planeación urbana, diseño urbano y arquitectónico, construcción y manejo de obras e intervenciones en el patrimonio. Investigadora del Grupo Gestión Integral del territorio - GIT, con experiencia en las líneas de investigación gestión del patrimonio y hábitat sostenible y solidario con el proyecto: "Escuela de Artes y Oficios del Parque temático Pueblito Nortesantandereano - Pamplona", desarrollado como trabajo de grado con calificación de excelencia y recomendación a meritorio.

Recibido: I de marzo de 201 I Aprobado: 26 de abril de 2011

Aspectos técnicos

Fuente: Reyes 2010 


\section{INTRODUCCIÓN}

En el trabajo se abordan varios momentos del saber, asociados a concepciones sobre desarrollo y cultura, que denotan la importancia de las relaciones ambientales, socioculturales y económicas en la construcción de la territorialidad; aproximaciones disciplinares de la arquitectura y el arte con los saberes y oficios, como fuente de procesos de fortalecimiento de identidades; estudio de los referentes culturales de las artes y oficios en el contexto específico de Norte de Santander y aspectos relacionados con las Escuelas de Artes y oficios y su pertinencia sociocultural para una región determinada. De esta manera, los principales resultados están representados en una propuesta para el diseño de una Escuela de Artes y Oficios en el municipio de Pamplona, con la cual se pretende contribuir al desarrollo sinérgico del territorio.

\section{METODOLOGÍA}

La estructura hermenéutica del proyecto se fundamenta en la interpretación de la acción social con significado subjetivo y en una secuencia que permite simultáneamente caracterizar y analizar signos; interpretar y descubrir su sentido expresado en símbolos de uso y apropiación territorial; modelar y comunicar por medio de la reconceptualización y recontextualización de saberes; retroalimentar con la confrontación de logros cognoscitivos y procedimentales y aplicar mediante un diseño que surge de la articulación de intereses ambientales y culturales.

\section{RESULTADOS}

\section{Identidades y territorialidad}

La noción actual de desarrollo aborda al territorio, no solamente como un espacio natural, sino también como un espacio que soporta relaciones del ser humano con la naturaleza, con su comunidad y su historia y relaciones de poder y de producción. Por lo tanto, los procesos de planificación deben responder a un enfoque integral dirigido a proteger y respetar el espacio natural "a partir del uso adecuado y de la apropiación consciente"'.

Desde este enfoque, se asume el carácter intangible del capital sinérgico como una propiedad emergente en la que adquieren gran relevancia los comportamientos, las costumbres y las tradiciones, así como los procesos de participación ciudadana y asociatividad de los habitantes "que reclaman el derecho a su propia memoria y a la construcción de su propia imagen" (...) en el marco de una "universalidad deshistorizada del progreso y de la homogenización que impone la modernización”

Según Rey ${ }^{3}$ La irrupción de la sociedad del conocimiento, la expansión de la información, el fortalecimiento de industrias culturales así como la importancia de una política de reconocimiento y la aparición de importantes movimientos socioculturales le han dado otro peso y otra significación a la presencia de la cultura en el desarrollo Así, al "abordar el territorio como resultado de las diferentes alternativas culturales que se realizan sobre un espacio físico de estipuladas condiciones socioambientales" ${ }^{4}$ se puede identificar que existen con-

\footnotetext{
MOSQUERA, Jemay. Planificación Ambiental y Arquitectura Sostenible. En: Revista Ambiental Agua, Aire y Suelo. Universidad de Pamplona, Colombia. Vol. I, No. 2, p. 92

2 MARTÍN-BARBERO, Jóvenes: comunicación e identidad. En: Pensar lberoamérica. Revista de cultura de la Organización de Estados Americanos (OEI), Barcelona, 2002.

3 REY, Germán. Cultura y Desarrollo Humano: Unas relaciones que se trasladan. En: Pensar Iberoamérica. Rev. de cultura de la Organización de Estados Americanos (OEI), Barcelona, 2002.

4 REYEZ, Celesky. Escuela de artes y oficios del parque temático pueblito norte santandereano - Pamplona. Trabajo de grado para optar por el título de arquitecta. Universidad de Pamplona, Pamplona. Colombia. 20I I. p. 17
} 
cepciones alternativas de la identidad - constructivista, esencialista e histórico-estructural ${ }^{5}$ las cuales ponen de manifiesto su valor sinérgico y su papel como eje fundamental del desarrollo integral del territorio ${ }^{6}$

\section{El papel de los hechos arquitectónicos en la historia}

Los procesos de diseño encuentran su principal fuente de inspiración en la forma como la especie humana interpreta la realidad (como seres individuales de pensamiento comunitario) y en la manera como las personas combinan la práctica y la teoría para apropiar, transformar y aprovechar el medio natural en el desarrollo de sus actividades cotidianas. Como fuentes de conocimiento, la práctica y la teoría, se rigen históricamente por saberes y conceptos que han permeado la enseñanza teórica de la arquitectura y los desarrollos teóricos de los hechos arquitectónicos ${ }^{7}$. Las características de estos conceptos están asociadas filosóficamente con el ser humano, la tecnología y la belleza/gusto/deleite y, a su vez, confluyen en el proyecto arquitectónico como síntesis de humano, lo tecnológico, lo ético, lo lógico y lo estético.

En el desarrollo del pensamiento arquitectónico se pueden diferenciar diversas etapas de su concepción. En el Renacimiento, se genera una transformación significativa en las actividades de diseño y construcción en tanto "los artesanos pudieron agregar (...) las nuevas invenciones surgidas durante la Edad Media; y (...) los intelectuales pudieron aportar (...) los métodos lógicos de argumentación (...) y los recién elaborados métodos de cálculo.”8

Durante los siglos XVIII y XIX, la Revolución Industrial permea los sistemas político, económico y social, y catapulta la actividad proyectual al considerarla como una manifestación histórica de la cultura estética occidental. En esta configuración y significación del desarrollo tecnológico, la arquitectura adquiere claras diferencias disciplinares, pero continúa mediada por la necesidad de aportar, en articulación con otras disciplinas, formas comunes de generación y transmisión de conocimiento.

En la segunda mitad del siglo $X X$ se rebate el enfoque newtoniano, que concebía al mundo como un mecanismo regido por leyes naturales inmutables y se sientan las bases para el surgimiento del paradigma de la complejidad y el Pensamiento Complejo. Esta gran transformación puso en evidencia la crisis del modelo económico globalizante y la crisis de una arquitectura formalista sin contexto ni personalidad, pero al mismo tiempo permitió reconocer a la arquitectura como un proceso creativo de múltiples factores y elementos interactuantes e interdependientes, con capacidad para asumir la impredecibilidad de los fenómenos, y conservar y fortalecer su individualidad, en el contexto espacio temporal en que estos se desenvuelven ${ }^{9}$

\section{Aspectos básicos de la Escuela de Artes y Oficios}

En los diferentes procesos de desarrollo, la práctica se ha convertido en un elemento que permite asumir los hechos arquitectónicos, las manifestaciones artísticas y las artesanías,

5 LARRAIN, Jorge. 1996. Modernidad, Razón e Identidad en América Latina. Edit. Andrés Bello, Santiago de Chile, p. 215

6 BOISIER, Sergio. El desarrollo territorial a partir de la construcción de capital sinergético. En: Estudios Sociales, No. 99 , CPU., Santiago de Chile, 1996. p. 16

7 Por ejemplo, los conceptos de "utilitas, firmitas y venustas" propuestos por el arquitecto Romano Vitruvio en el siglo I d.C., "soliditas, comoditas y voluptas" propuestos a finales del siglo XV por el arquitecto italiano León Battista Alberti o conceptos contemporáneos de tectónica, fenomenología, espacialidad y operatividad.

8 BERNAL, John. La ciencia en la historia. México, U. Nacional Autónoma de México, 1972.

9 MOSQUERA, Jemay. Arquitectura y complejidad. En: Revista Ambiental Agua, Aire y Suelo. Universidad de Pamplona, Vol. 2, No. I, año 2, Pamplona, Colombia, 2007. p. 8. 
como objetos de deleite estético y como instrumentos socialmente útiles. Otro elemento relevante es el fenómeno artístico, cuyo proceso comienza con la elaboración mental de la obra y continúa, a través de la técnica, en la forma como la idea se plasma en materia.

La transformación de los materiales y la técnica en el tiempo pueden ser definitorios de un determinado lenguaje o estilo artístico. Por lo tanto, es primordial tener en cuenta las interacciones de la arquitectura y el arte con diversos elementos (estética, fenomenología, comunicación, normativa y economía, entre otros), cuyo sentido y peso relativo varía de acuerdo a las condiciones y reivindicaciones sociales de cada periodo histórico.

En lo que respecta al hecho arquitectónico Habermas plantea el Proyecto Moderno como un proyecto inacabado, no superado, un proyecto con ideales sociales pendientes y diferentes en cada contexto, perspectiva que permite abordar los interrogantes: ¿Cómo debe vivir el ciudadano del común? -Y: ¿Cómo desarrollar la función social de la arquitectura? ${ }^{10}$ Así las cosas, dentro de las edificaciones que pueden contribuir a los procesos de valoración y recuperación de los saberes tradicionales, se encuentran las Escuelas de Artes y Oficios. En ellas se articula la ciencia y la inteligencia en acciones que demandan diversidad de actividades con énfasis artesanal, industrial o combinado. Producto de las manifestaciones que las comunidades de cada contexto apropian y aplican para la construcción de la territorialidad, los trabajos manuales que se desarrollan en las Escuelas de Artes y Oficios son diversos y generalmente se relacionan con carpintería, forja, cerámica, bordados, cestería y joyería.

Es importante recordar que las artes están a la altura de las profesiones científicas, se entienden como " el conjunto de reglas o preceptos que se tienen en cuenta para hacer bien alguna cosa" "y son fundamentales para la gestión del conocimiento, pues subyacen en las ideas, valores, signos y símbolos de poblaciones concretas. En el desarrollo de las artes, la actividad productiva artesanal se fundamenta en tres factores clave, el producto, el taller artesanal (espacio de ejecución) y el oficio (espacio estructural que posibilita la actividad ordenada, material y lógicamente). Por su lado, la unidad de producción articula el taller y la producción de los talleres del mismo oficio; cumple la función múltiple e interactiva de creación, enseñanza y organización; y es el marco de referencia del proceso de realización del individuo como oficiante productor en un oficio que implica destreza técnica y sensibilidad estética.

Con el desarrollo social y el intenso contacto intercultural en el medio urbano, la producción artesanal ha exigido cualificación en aspectos de diseño y de expresión estética, ya que: "Las formas objetivadas o materializadas sólo cobran sentido si pueden ser apropiadas y permanentemente reactivadas por sujetos dotados de "capital cultural incorporado", requerido para "leerlas", interpretarlas y valorizarlas" 12 Es así como las Escuelas de Artes y Oficios renuevan su importancia, pues ofrecen conocimientos técnicos asociados a las identidades culturales locales, los cuales contribuyen a la definición de estilos personalizados y aportan a la formación de comunidades capaces de incrementar sus ingresos y mejorar su calidad de vida.

Mientras que en Europa, las escuelas de artes y oficios medievales perdieron vigencia, en Latinoamérica se crean, desde mediados del siglo XIX, las primeras Escuelas de Artes, las cuales fueron reconocidas como sinónimo de progreso e integración social, ya que no sólo

10 HABERMAS, Jürgen. El Discurso Filosófico de la Modernidad. Katz Editores, Argentina, 2008.

II URDANETA, Alberto. Apertura del curso de dibujo natural en la Universidad Nacional. En: Anales de la Universidad Nacional de los Estados Unidos de Colombia. Vol. 3, No. 18; (s.c), 1870. p. 409

12 GIMÉNEZ, Gilberto. Territorio, cultura e identidades. En Globalización y regiones en México, México, Coord. Rosales, Programa Universitario de Estudios sobre la Ciudad, UNAM/ Porrúa, 2007, p. 28. 
representaban aprendizaje, sino también la generación de nuevos elementos que respondían a la reinterpretación de culturas ancestrales. De esta forma, la expansión del sistema de fábrica que se dio en la primera mitad del siglo $X X$, no logró invalidar los oficios manuales, ni las experiencias y destrezas ya adquiridas por parte de obreros y pequeños manufactureros en el trabajo de la industria. Entre las escuelas fundadas en Colombia entre 1860 y 1940, en las que se evidencia la presencia de oficios tradicionales, modernos y mixtos, vale la pena destacar el Instituto Técnico Central: una Escuela de Artes y Oficios dirigida por comunidades cristianas y filantrópicas para el "regeneramiento" de niños y niñas de la calle. Además, se observa la existencia de "talleres privados en los que la enseñanza de artes y oficios descollantes eran organizados en torno a la figura del maestro, estaban desprovistos de control estatal, y eran coordinados por artesanos con características universales de maestros medievales o coloniales: hombres, generalmente de formación religiosa, que contribuyeron en gran manera al perfeccionamiento de las artes y oficios y conservaron, por ello, su longevidad como sujetos de prestigio y dignidad tras años de labores. ${ }^{13}$

En el Nuevo Reino de Granada no existía una instrucción pública sino gentes e instituciones, (la iglesia, las encomiendas, los resguardos, la familia) que se dedicaban a la "transmisión que permitía a un individuo asimilar el alfabeto y los números" "4Paralelo a la Instrucción Pública que se inicia con la expulsión de los jesuitas en 1867 , funcionaban otros espacios educativos como los resguardos, las parroquias, las Escuelas de Artes y Oficios, que ofrecían preparación para el trabajo y para la vida.

La Constitución Política de Colombia de 1886 representa una reforma que declara la gratuidad (más no obligatoriedad) de la enseñanza oficial y promueve la creación de las primeras Escuelas de Artes y Oficios. Luego, la ley I 2 I de I 887 dispone la creación de varias Escuelas de Artes y Oficios en el país, con destino a la preparación adecuada de los artesanos y la ley 48 de 1892 ordena la creación de Escuelas de Artes y Oficios en el Guamo, en Panamá y Pasto, como respuesta a la tradición productiva manufacturera de estos sitios, de tal forma que para los años treinta del siglo pasado ya existían en Nariño Escuelas de Artes y Oficios en Túquerres, Ipiales, Ancuya y Pasto, con talleres de mecánica, electricidad, carpintería, ebanistería, tapicería, fotografía y tejidos, entre otros ${ }^{15}$.

Cabe destacar, que la Ley 39 de 1903 sobre instrucción pública reglamenta la posibilidad de fundar y sostener Escuelas de Artes y Oficios, en las cuales se enseñe artes manufactureras y especialmente el manejo de máquinas aplicables a las pequeñas industrias, talleres para la enseñanza gratuita de artes y oficios que, según las necesidades, las condiciones y las costumbres de la respectiva localidad, convenga difundir de preferencia en ella (por ejemplo, el Instituto San Antonio de Bogotá, enfocado a la enseñanza teórica y práctica de agricultura y artes y oficios mecánicos a niños pobres. ${ }^{16}$

Como se puede observar, desde fines del siglo XIX, las Escuelas de Artes y Oficios jugaron un rol fundamental en la instrucción de mano de obra calificada, se consideran el precedente más representativo de la formación técnica colombiana y han contribuido notablemente a la recuperación de saberes tradicionales, a la capacitación de grupos de jóvenes provenientes de segmentos de población vulnerable y a la recuperación de edificaciones patrimoniales y bienes de interés cultural.

\footnotetext{
PORTILLA, Portilla, Isabela. 20I I. Disponible en: http://www.javeriana.edu.co/revistas/ Ofi/pesquisa/wordpress/?p=34 I HELG, Aline. La educación en Colombia. 1918-1957. CEREC. Bogotá. 1987.

MUÑOZ, Lydia Inés, et al Historia del Instituto Técnico Industrial: desde la escuela de artes y oficios: 1931-2007. Pasto. 2007. p. 16

16 BETANCUR, Gabriel. "Presentación Ley 39 de 1903” En: Revista Colombiana de Educación. No. 13. 1984
} 
Actualmente, el Ministerio de Cultura reconoce la experiencia de las Escuelas Taller de Bogotá, Cartagena, Mompox, Popayán, Salamina y Barichara, creadas desde 1992 por la Agencia Española de Cooperación Internacional para la salvaguardia del patrimonio cultural y la formación en oficios a la población vulnerable. Mediante el Programa Nacional Escuelas Taller Colombia, herramientas para la paz, el Ministerio de Cultura propende por el reconocimiento y el respeto por las diferencias culturales y busca fortalecer capacidades locales para el desarrollo social y económico, a través de tres principios: i) Valoración, apropiación y salvaguardia del patrimonio cultural, ii) Enfoque diferencial, acciones afirmativas y sin daño a las comunidades locales $y$ a las poblaciones vulneradas o vulnerables, y iii) Emprendimiento e industrias culturales y creativas. $^{17}$

\section{Artes y oficios en Norte de Santander}

En Colombia, las normas y leyes vigentes, la Constitución Política de 1991, la ley 388 de 1997 y la ley I 454 de $20 \mathrm{I}$ I, cuentan con postulados culturales, socio-ambientales, políticos y económicos que promueven la autonomía, la descentralización y las articulaciones locales. No obstante, la multidimensionalidad de las transformaciones globalizantes externas y los conflictos internos, contribuye al aumento de la fragmentación territorial y al deterioro de las estructuras urbanas.

Según la Ley 36 de 1984 y el Decreto 258 de 1987, la artesanía es una actividad de transformación para la ejecución de un producto específico que cumple una función decorativa o utilitaria; se realiza con predominio de energía humana (física y mental), herramientas y máquinas relativamente simples; y es condicionado por el medio ambiente físico y el desarrollo histórico cultural. Se pueden diferenciar varias clases de artesanías tales como, la autóctona aborigen, la de proyección aborigen, la tradicional, la típica folclórica, la urbana y la suntuaria ${ }^{18}$ (las cuales son determinadas por el medio ambiente, el hábitat y la tecnología y están relacionadas con las manifestaciones culturales de comunidades nativas, las manifestaciones especializadas de carácter anónimo y las manifestaciones culturales procedentes de diferentes culturas.

Según Artesanías de Colombia, 350.000 personas producen artesanías en el país, lo que corresponde al $15 \%$ de la industria manufacturera ${ }^{19}$ Sus manifestaciones culturales expresan la variedad étnica, religiosa, de costumbres y tradiciones, así como la riqueza y diversidad natural de los diferentes territorios nacionales. Entre los productos naturales que ofrece el territorio, se destacan, la arcilla, el bambú, el cuero, las fibras, el fique, la guadua, la lana, la madera, la tagua, el totumo y los metales preciosos, entre otros. Como resultado de su transformación, se producen hamacas, piezas de oro, filigranas en oro y plata, cestería, tapicería, cerámica, accesorios en coco o semillas, bordados, muebles, esculturas en tagua, objetos de madera y artículos tejidos a mano, los cuales representan a Colombia en el mundo.

Si bien, el escenario en el que se desenvuelve la sociedad de Norte de Santander y del municipio de Pamplona (Figura I) proporciona las bases de las identidades locales, existen diversos factores que atentan contra los componentes tangibles e intangibles que las conforman y que actúan en contra de la imagen y la memoria colectiva construida por varias generaciones. No obstante, el potencial cultural y educativo de Pamplona, la fragmentación

17 MINCULTURA, 20II. http://vigias.mincultura.gov. co/?p=855

I8 Instituto Interamericano de Cooperación para la Agricultura (IICA). Metodología para construir perfiles de peligro fitosanitario de plantas, productos vegetales y otros artículos reglamentados. San José, CR. 2009.

19 PROEXPORT. Promoción del turismo, inversión y exportaciones - http://www. colombiaespasion.com/es/sala-de-prensa/73contenido-prinicipal/750-colombia-un-pais-de-artesanos.html 
social ha ganado espacio y se han perdido los espacios de representación cultural, de tal manera que se requiere la optimización de la infraestructura de servicios educativos y el fortalecimiento del rol de los actores locales en la definición de alternativas apropiadas de uso y ocupación del territorio.

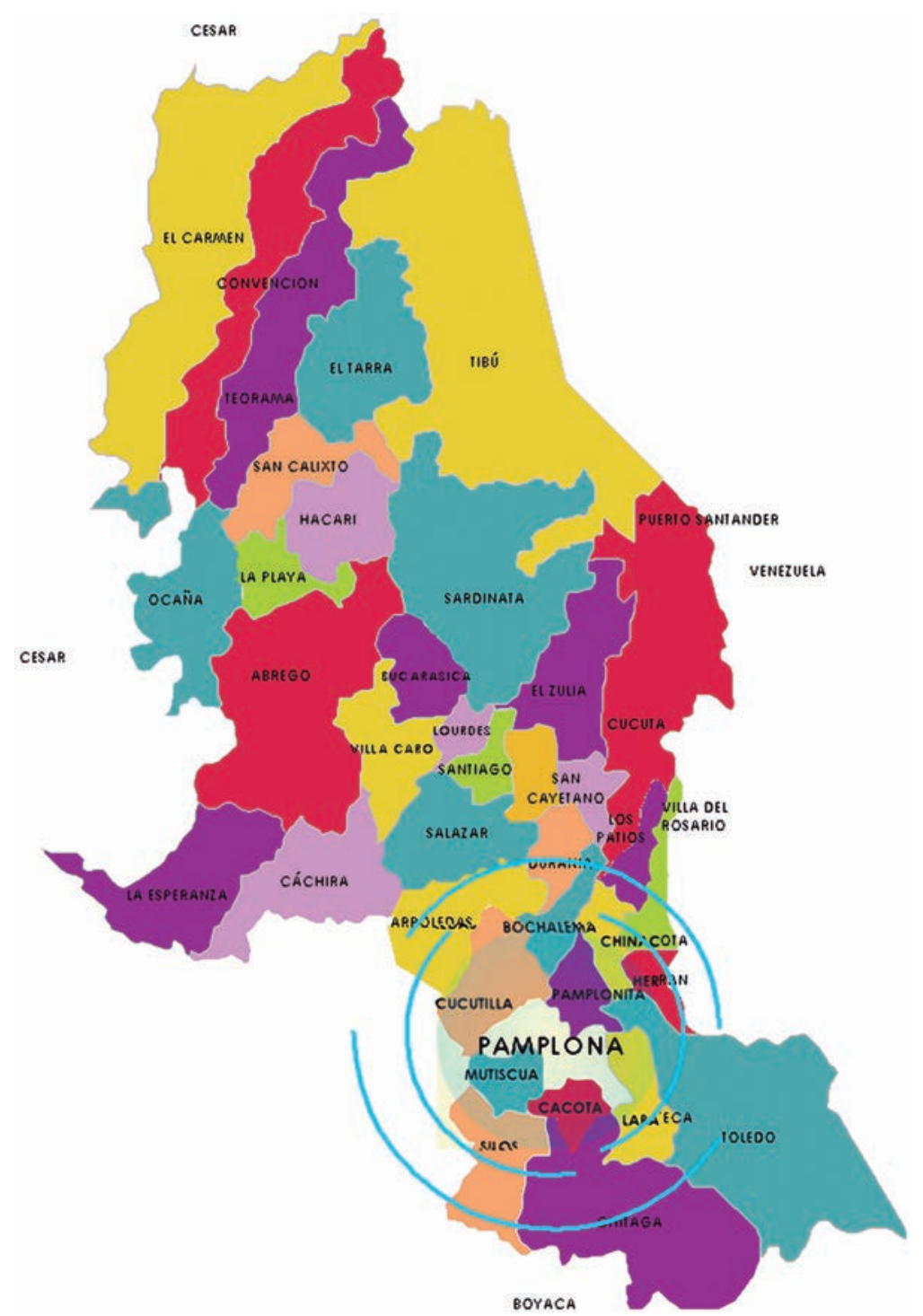

A partir del estudio desarrollado sobre la base Sistema Nacional de Información Cultural (SINIC) del Ministerio de Cultura de Colombia, se identificaron las principales dinámicas de las artes y oficios en dieciocho Municipios de Norte de Santander: Bochalema (enjalmas y tejidos), Bucarasica (cestería), Cáchira (tejidos), Cácota (tejidos en lana virgen, materos y jarrones de arcilla), Chitagá (trabajos en lana virgen, canasto de cañato), El Tarra (objetos en tronco de plátano), Lourdes (cestería en bejuco, tejido de telar rústico), La Playa de Belén (cerámica), Mutiscua (tallas en mármol y trabajos en lana), Ocaña (bordados, escultura y joyería), Pamplona (bordados), Salazar (escobas de lucua tejida y tallado en piedra), Santiago (talla en madera), San Cayetano (cestería en bejuco), Sardinata (talla en madera, tejidos en nylon y esteras), Silos (trabajos en lana y telar, tamas de esparto), Tibú (cestería de la comunidad motilón Bari, objetos de caza Bari y bajo relieves en totumo), Villa del Rosario (escultura, mimbre y bambú, ratán (talla en Madera). Dichas dinámicas fueron representadas en cada municipio (Figuras 2, 3, 4 y 5)
Figura I. Ubicación de Pamplona en Norte de Santander

Fuente: Reyes, 2010

\section{129}


Figuras 2, 3, 4 y 5. Artesanías en municipios de Norte de Santander

Fuente: Reyes, 2010

Figura 6. El tejido como eje focalizador de las Artes y oficios del departamento

Fuente: Reyes, 2010
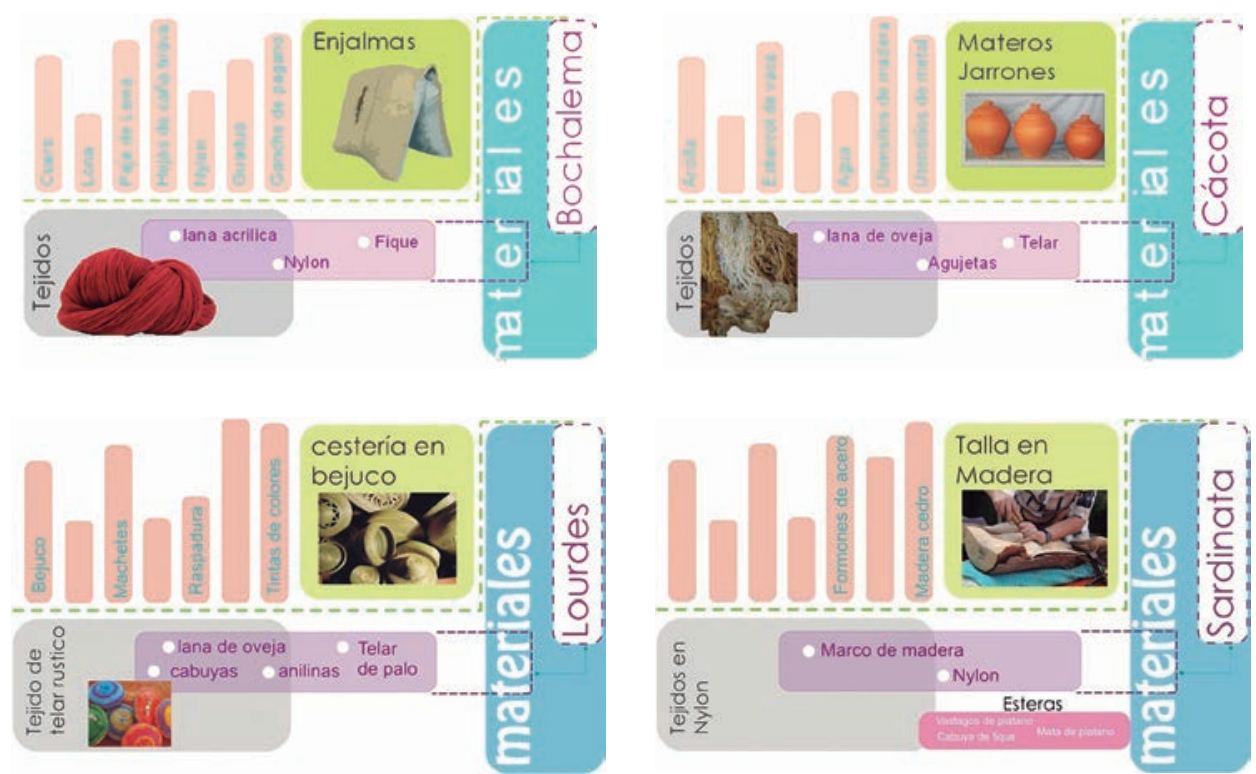

Según el estudio de las dinámicas artesanales, en quince de los dieciocho municipios analizados se evidencia el desarrollo de tejidos con fibras naturales flexibles (lana) y fibras semirrígidas (bejuco y vástago del plátano), cuya manifestación es muy remota e inicia en la prehistoria indígena con la capacidad ancestral de hilar una fibra, entrelazar los hilos, formar una urdimbre y producir una superficie textil homogénea. Posteriormente, al proceder a su decoración por medio de la pintura, del bordado o de la intromisión de hilos de variados colores y materias, nace el actual tejido artístico que se asume como referente cultural de las artesanías del departamento (Figura 6).

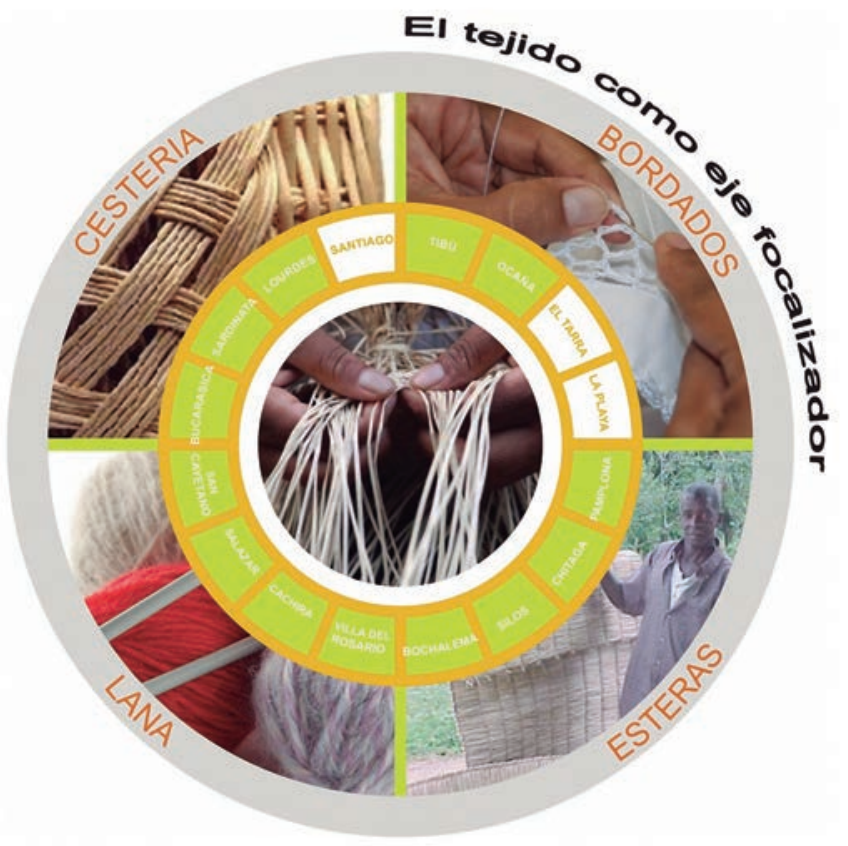

Proceso de diseño de la escuela de artes y oficios

Habida cuenta que "el concepto de arquitectura está en la unión y en la colaboración entre las artes $(. .$.$) y es el conjunto de modificaciones y alteraciones introducidas en la superficie$ 
terrestre con objeto de satisfacer las necesidades humanas". ${ }^{20}$ se plantea la afinidad entre la arquitectura, las artes y las artesanías mediante la aplicación de conceptos básicos comunes para solucionar las necesidades propias de la materialización del objeto deseado, su imagen, uso, estructura, espacialidad y belleza.

Producto del análisis de las dinámicas artesanales locales, se asume la interpretación del tejido como base conceptual y metafórica para la configuración de la lógica de diseño de la escuela de artes y oficios de Pamplona. El estudio de los oficios predominantes en el departamento permitió la definición de los talleres de la escuela de artes y oficios (cestería, bordados y tejidos, alfarería y ebanistería), los cuales se proponen con el fin de rescatar, mantener y potencializar dichos saberes (Figura 7).

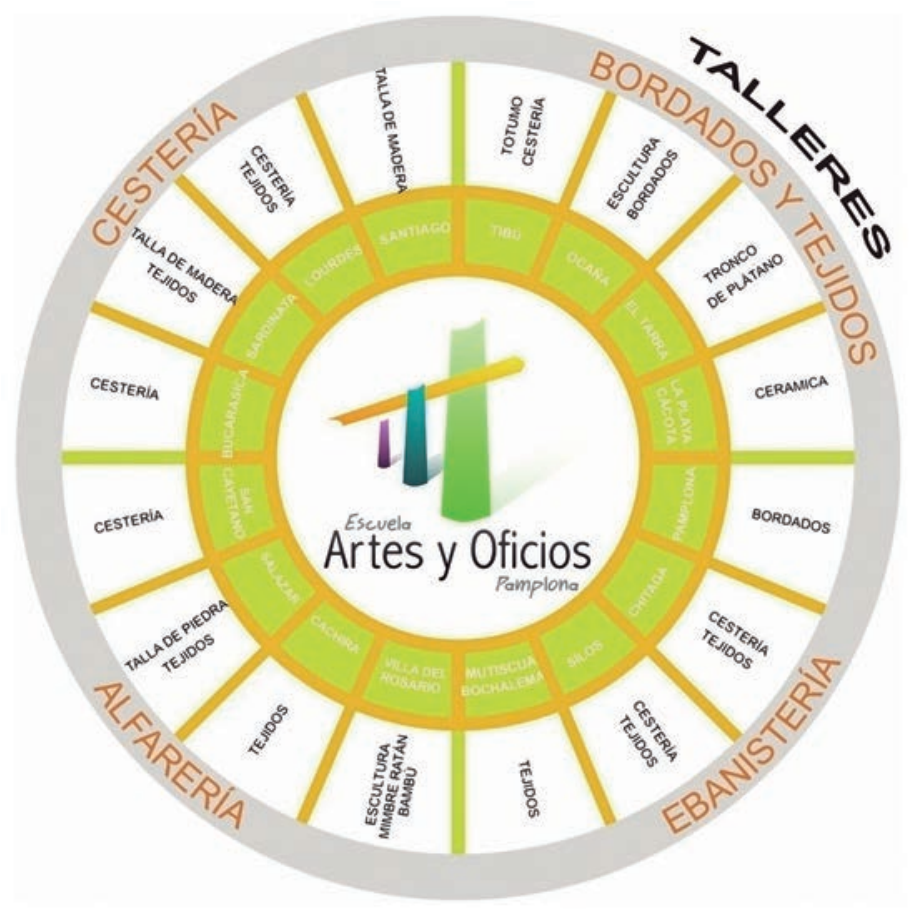

Adicional a la conceptualización inicial y los procesos enunciados de conformación de Escuelas de Artes y Oficios en Colombia, en el desarrollo del proyecto, se analizaron referentes como, el Centro Cultural y Educativo Leonardo Fabio del Municipio de Lanús, en Buenos Aires, Argentina y La Nueva Escuela de Arte de Zaragoza, en España, lo que permitió identificar sus principales características en cuanto a materialidad y espacialidad, tectónica, operatividad y fenomenología, como base para la definición y recontextualización de los parámetros de diseño de la escuela de artes y oficios para Pamplona.

La contextualización del proyecto responde a la ubicación de Pamplona a $2642 \mathrm{msnm}$, a su temperatura promedio de $10^{\circ} \mathrm{C}$ y a las condiciones de un lote urbano de $1500 \mathrm{~m}^{2}$ ubicado en la parte posterior del colegio Provincial San José, caracterizado por pendientes suaves que generan un desnivel de $5.00 \mathrm{~m}$ y por un entono suburbano verde cuyo paisaje relativamente quebrado no se encuentra espacialmente relacionado con el centro histórico de la ciudad. Lo anterior, permitió realizar una aproximación fenomenológica y, a partir de la metáfora, lograr una exploración consecuente con las condiciones jerárquicas del tejido y la urdimbre definidas anteriormente.
Figura 7. Talleres establecidos Fuente: Reyes, 2010

20 PATTETA, Luciano. Historia de la Arquitectura. Madrid: Celeste Ediciones. 1997.p. 71

\section{131}


En el proceso de exploración de las aplicaciones de las técnicas de tejidos artesanales se buscó emular e interpretar un tejido base de urdimbre-trama y se pudo llegar al planteamiento de la imagen inicial del proyecto (Figuras 8,9 y 10 ).

Figuras 8,9 y 10. Exploraciones

Fuente: Reyes, 2010
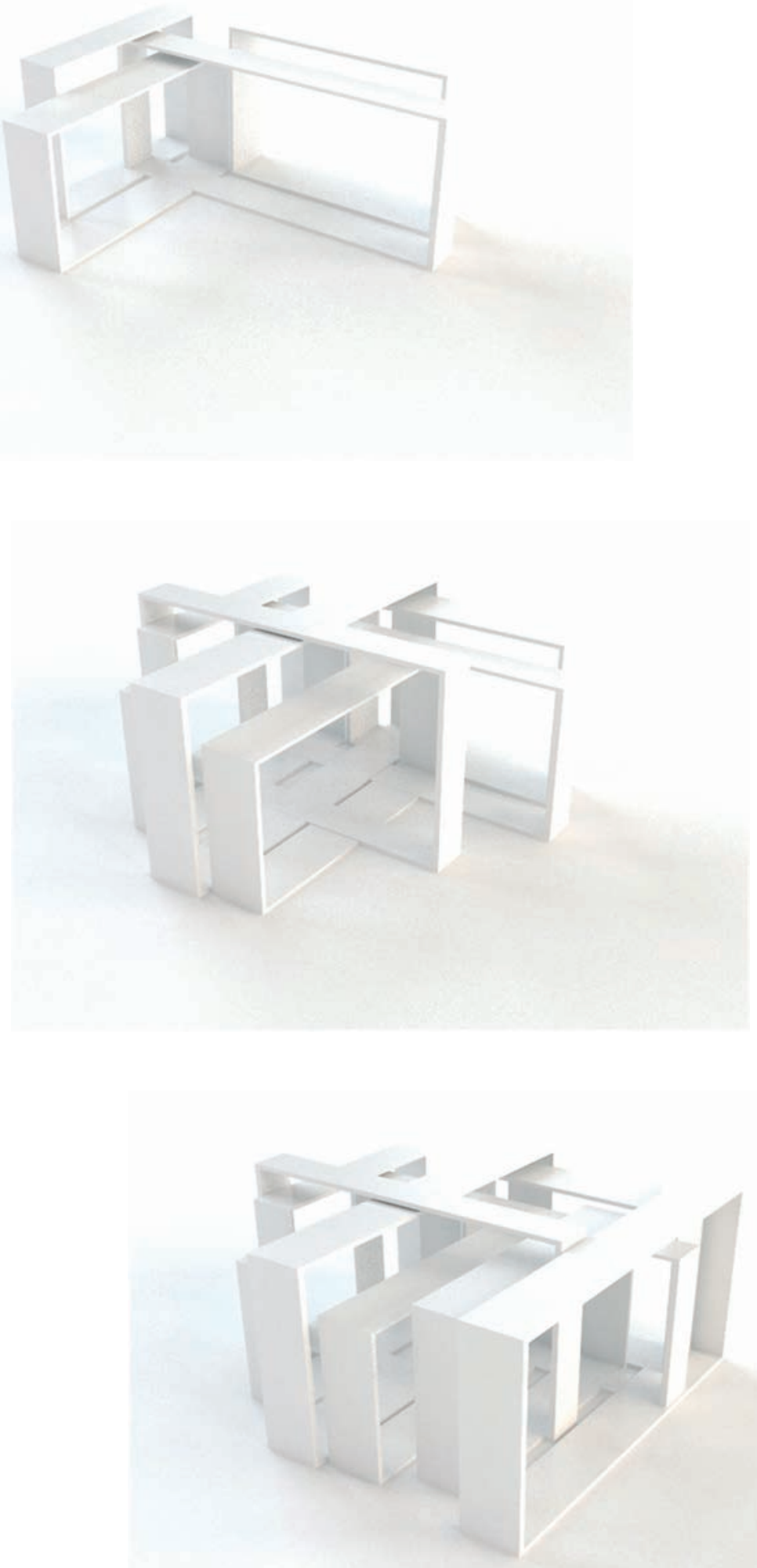
La imagen y su correspondiente programa arquitectónico, fueron complementados con aspectos de normatividad urbana, implantación urbanística, aproximación estructural, organización espacial, relaciones espaciales, aspectos programáticos y simbólicos, los cuales permitieron llegar a la formulación del diseño de la Escuela de Artes y Oficios de Pamplona (Figuras II, 12, 13 y 14).
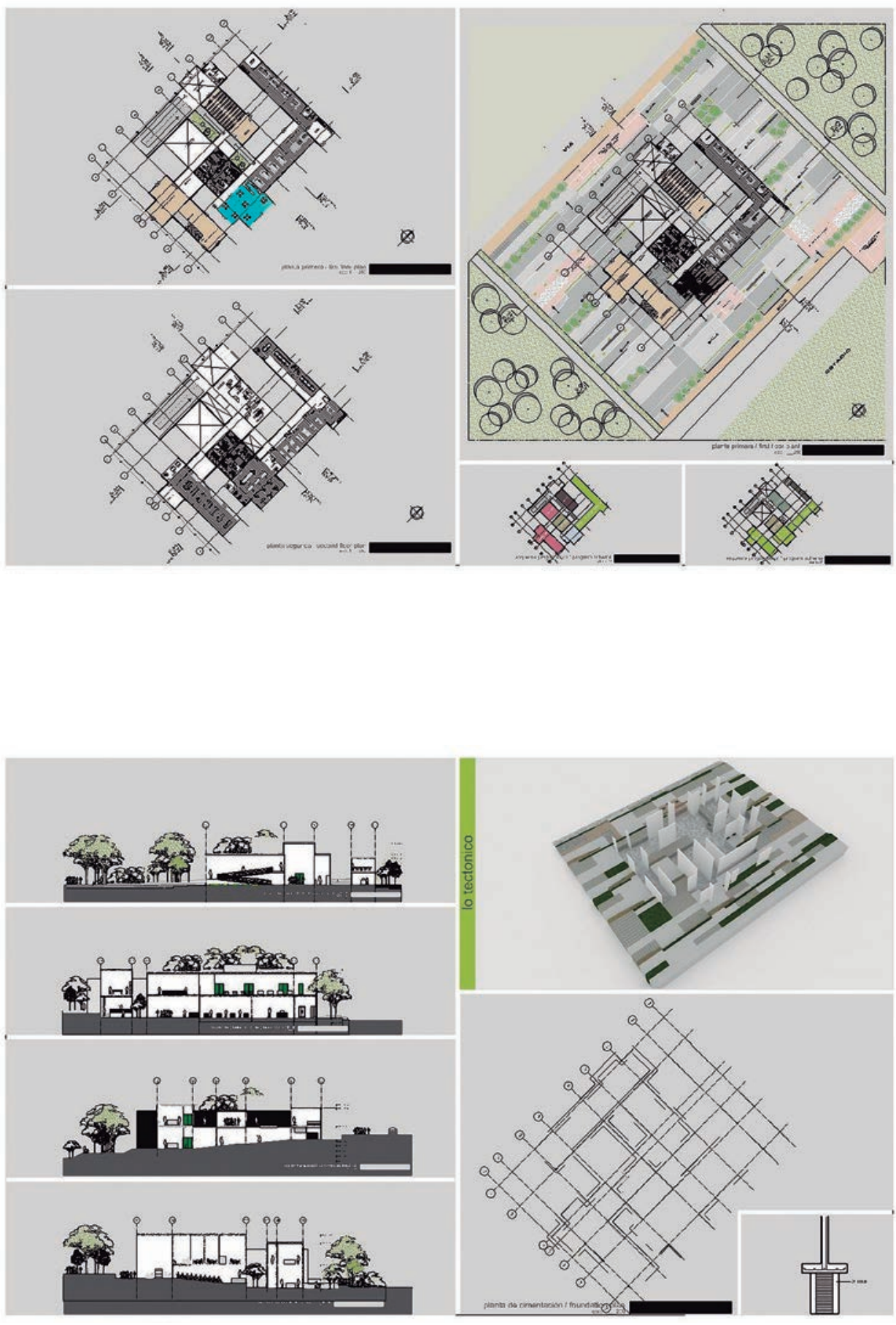

Figura I I. Plantas Arquitectónicas I y 2 pisos Fuente: Reyes, 2010

Figura 12. Aspectos técnicos Fuente: Reyes, 2010 


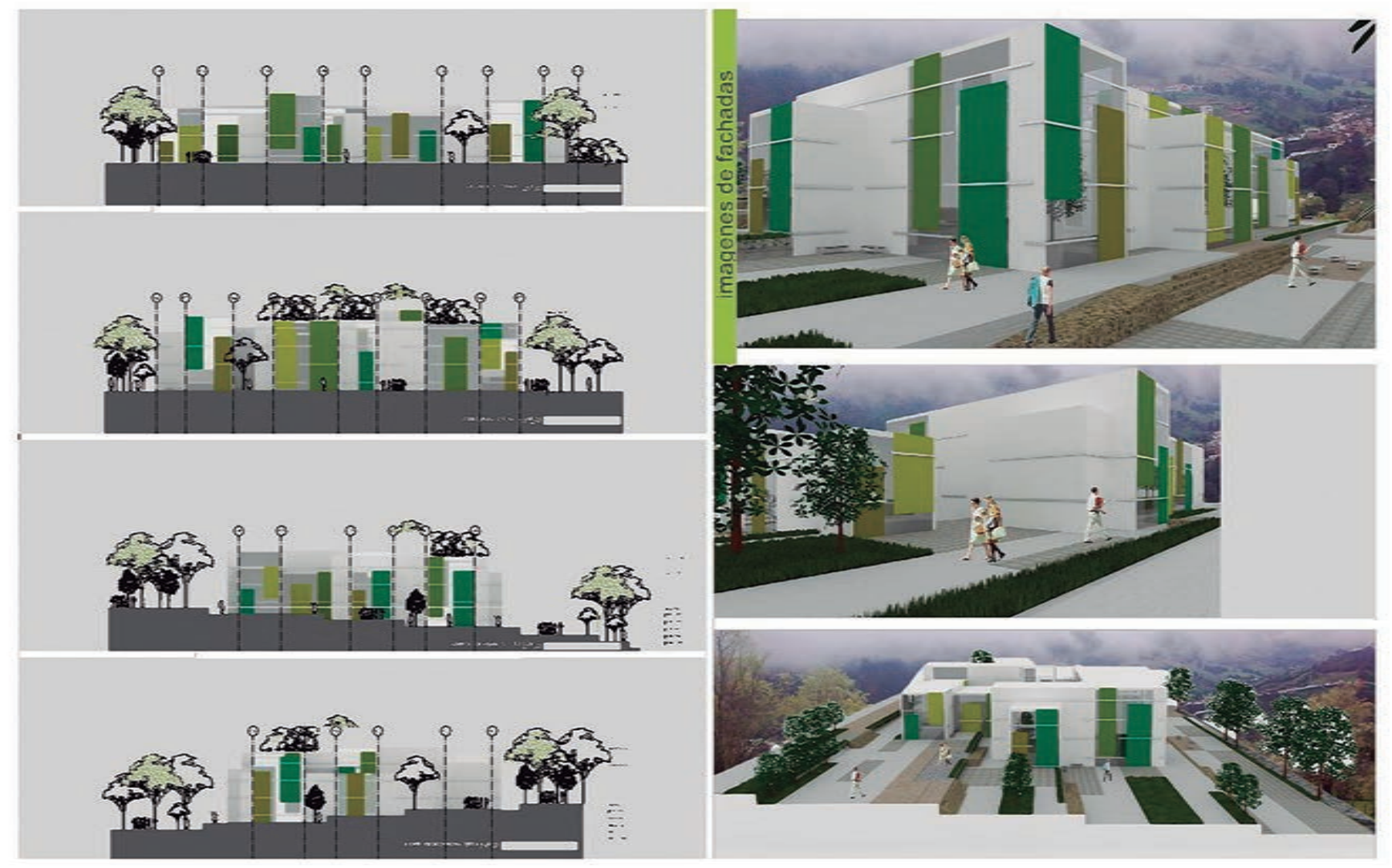

Figura 13. Alzados e imágenes

Fuente: Reyes, 2010

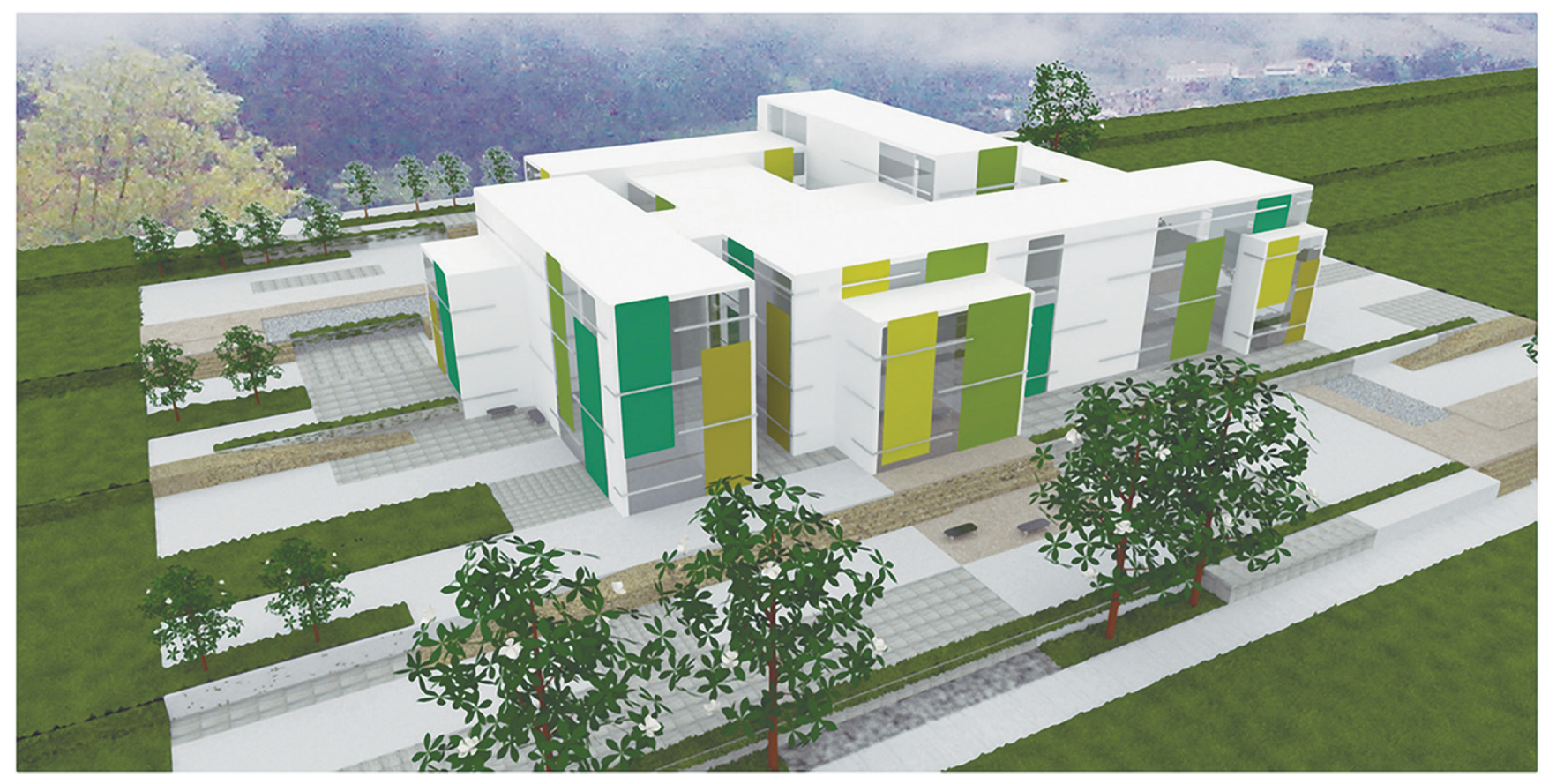

Figura 14. Proyecto Escuela de Artes y Oficios

Fuente: Reyes, 2010 
A continuación, se relacionan algunos aspectos que representan un balance entre las características ambientales y culturales del entorno con las necesidades operativas de la edificación:

- Configuración simbólica de la edificación, ambientes, espacios y formas arquitectónicas, a partir de la abstracción del tejido como base para dar solución a los diferentes aspectos de diseño.

- Articulación del espacio público con el sistema de patios, circulaciones y revestimientos para reflejar la génesis del tejido en el proyecto. Las franjas verdes que simulan la trama y la urdimbre, se conectan entre sí por todo el terreno y se adaptan a los cambios de nivel mediante texturas en el espacio público y acabados de fachada en la edificación.

- Adaptación al contexto del colegio existente en el predio, al proceso de diseño del parque temático en el lote contiguo y a la topografía del lugar por medio de niveles, rampas y un sistema de circulaciones, que aseguran la libre accesibilidad y movilidad de las personas, configuran los espacios al aire libre $y$ articulan los diferentes componentes del proyecto.

- Uso de materiales propios de la región (ladrillo y elementos decorativos en arcilla) para ahorro de recursos en términos de producción y apropiación cultural del entorno.

- Aplicación de materiales tradicionales (acero, concreto, ladrillo y vidrio) en un sistema de cimentación de zapata corrida reforzado con pilotes in situ que responde a un alto nivel freático del lote; un sistema estructural de muros portantes con losas aligeradas que brinda flexibilidad en la configuración espacial del diseño y menos requerimientos de herramienta y mano de obra especializada; $y$ un sistema de revestimiento en muros de ladrillo y grandes ventanales que genera transparencia visual y un fácil reconocimiento espacial del proyecto.

- Aprovechamiento de la luz natural con ventanales de doble vidrio hermético y celosías en polipropileno de alta densidad que aseguran eficiencia lumínica.

- Disposición de elementos de protección solar y control térmico (sistema de patios para la generación de corrientes de aire y ventanales que actúan como diafragmas de control termoacústico y de transición interior-exterior).

- Protección acústica en las divisiones de los talleres con muros en madera, plástico y lana aislante, que ayudan a reducir el ruido entre uno o varios talleres y brindan privacidad para laborar o realizar exposiciones.

- Articulación de las variables tangibles (producción, productividad, antropometría y ergonomía) e intangibles (confort, estética y espiritualidad) de diseño en la distribución operativa y organización espacial de los talleres, la zona administrativa, los sitios de esparcimiento, las salas de exposición y los espacios polivalentes.

\section{CONCLUSIONES}

Se requiere la adecuación de medidas legales y de concienciación sobre la importancia que el patrimonio adquiere como parte integrante de la memoria de nuestra civilización al dar a conocer nuestro modo de vida a las futuras generaciones. 
La arquitectura debe apoyarse en estrategias flexibles y adaptativas que permitan aprovechar los procesos culturales locales y conseguir un equilibrio entre los múltiples factores del contexto que inciden en un proyecto.

La técnica del tejido no es ajena a la técnica de diseño proyectual, pues consta de las mismas estructuras, principios básicos, partes y funciones de un diseño arquitectónico. La interpretación no sólo responde a la forma o imagen del elemento, sino también a las condiciones del entorno, como el clima y la cultura.

El diseño de una escuela de artes y oficios debe responder a criterios de sostenibilidad en el uso efectivo de recursos naturales y energéticos; de adaptabilidad a las interacciones de los componentes de la edificación y su entorno; de durabilidad acorde con la necesidad de seguridad de las personas; de confort antropométrico y de movilidad para los usuarios; $y$ de territorialidad acorde a los valores e identidades culturales locales.

\section{BIBLIOGRAFÍA}

BERNAL, John. La ciencia en la historia. México, U. Nacional Autónoma de México, 2a ed., 1972.

BETANCUR, Gabriel. "Presentación Ley 39 de 1903" en: Revista Colombiana de Educación. No. 13. 1984

BOISIER, Sergio. El desarrollo territorial a partir de la construcción de capital sinergético. Estudios Sociales, No.. 99, CPU., Santiago de Chile, 1996.

COLOMBIA. Ministerio de Cultura (Mincultura) 20I2. http://vigias.mincultura.gov. $\mathrm{co} / \mathrm{p}=855$

COLOMBIA. Promoción del turismo, inversión y exportaciones - Proexport http://www. colombiaespasion.com/es/sala-de-prensa/73-contenido-prinicipal/750-colombia-un-pais-deartesanos.html

GIMÉNEZ, Gilberto. “Territorio, cultura e identidades”. En Globalización y regiones en México, México, Coord. Rosales, Programa Universitario de Estudios sobre la Ciudad, UNAM/ Porrúa, 2007.

HABERMAS, Jürgen. El Discurso Filosófico de la Modernidad. Katz Editores, Argentina, 2008. 
HERRERA, Neve. Artesanía y Organización Social de su Producción. Artesanías de Colombia S.A. . 1992.

INSTITUTO INTERAMERICANO DE COOPERACIÓN PARA LA AGRICULTURA (IICAMetodología para construir perfiles de peligro fitosanitario de plantas, productos vegetales y otros artículos reglamentados. IICA. San José, C.R., 2009.

LARRAIN Jorge. Modernidad, Razón e Identidad en América Latina. Edit. Andrés Bello, Santiago de Chile, 1996.

MARTÍN-BARBERO, Jesús. Jóvenes: comunicación e identidad. Pensar Iberoamérica. Revista de cultura de la Organización de Estados Americanos (OEI), Barcelona, 2002. http://www. oei.es/pensariberoamerica/ric00a03.htm

MOSQUERA, Jemay. Planificación Ambiental y Arquitectura Sostenible. Revista Ambiental Agua, Aire y Suelo. Universidad de Pamplona, Colombia. Vol. I, No. 2, 2006.

MOSQUERA, Jemay. “Arquitectura y complejidad”. En: Revista Ambiental Agua, Aire y Suelo. Universidad de Pamplona, Colombia. Vol. 2, No. I, año 2. 2007.

MUÑOZ Lydia Inés, GUERRERO José Eduardo, Guzmán María Luisa et al Historia del Instituto Técnico Industrial: desde la escuela de artes y oficios: 193 I-2007. Pasto. 2007.

PATTETA, Luciano. Historia de la Arquitectura. Madrid: Celeste Ediciones 1997.

REY, Germán. "Cultura y Desarrollo Humano: Unas relaciones que se trasladan. Pensar Iberoamérica”. En: Revista de cultura de la Organización de Estados Americanos (OEI), Barcelona, 2002. http://www.oei.es/pensariberoamerica/ric00a04.htm

Panoramica de Pamplona

Fuente: Suministrada por los autores

REYES, Celesky. Escuela de artes y oficios del parque temático pueblito norte santandereanoPamplona. Trabajo de grado para optar por el título de arquitecta. Universidad de Pamplona. Pamplona. Colombia, 20II.

SALDARRIAGA, Alberto. Aprender Arquitectura. Un Manual de Supervivencia. Colombia. Ed. Fundación Corona, 1996.

URDANETA, Alberto. "Apertura del curso de dibujo natural en la Universidad Nacional". En: Anales de la Universidad Nacional de los Estados Unidos de Colombia. Vol. 3, No. I8; 1870. 